\title{
Coeficientes de Conversão do Kerma no Ar Incidente para a Dose Glandular Média em Mamografia de Mulheres com Implantes Mamários: Estudo Preliminar
}

\author{
Conversion Coefficients from Incident Air Kerma to Mean Glandular Dose \\ in Mammography of Women with Breast Implants: Preliminary study
}

\author{
Alexander C. F. Silva1,2, Josilene C. Santos ${ }^{2}$, Lilian S. Couto ${ }^{4,5}$, João E. Peixoto ${ }^{2,3}$ \\ ${ }^{1}$ Fundação Técnico-Educacional Souza Marques - FTESM, Rio de Janeiro, Brasil \\ ${ }^{2}$ Instituto de Radioproteção e Dosimetria - IRD/CNEN, Rio de Janeiro, Brasil \\ ${ }^{3}$ Instituto Nacional de Câncer José Alencar Gomes da Silva - INCA, Rio de Janeiro, Brasil \\ ${ }^{4}$ Clínica São Marcelo, Goiânia, Brasil \\ ${ }^{5}$ Programa de Mastologia, Universidade Federal de Goiás, Goiânia, Brasil
}

\begin{abstract}
Resumo
Na mamografia de mulheres com implantes mamários, além das quatro incidências padrões, são necessárias mais quatro com o deslocamento posterior do gel de silicone. Nesta prática radiográfica, a grandeza dosimétrica relacionada ao risco de câncer radioinduzido na mama é a dose glandular média $\left(D_{G}\right)$, obtida a partir do kerma no ar incidente $\left(K_{i}\right)$ e do coeficiente de conversão dose glandular normalizada $(D g N)$ entre estas grandezas. Este trabalho teve como objetivos: (a) investigar o comportamento do coeficiente $D g N$ em mamas de diversas espessuras com implantes de volumes variados por meio do software MCNPX; e (b) avaliar o impacto dos novos coeficientes $D g N$ na estimativa da $D_{G}$ para a mamografia de uma amostra de 40 mulheres com implantes. Para tal, foram simuladas mamas com e sem implantes, compostas de uma mistura homogênea de $50 \%$ tecido adiposo e $50 \%$ tecido glandular envolta em uma camada de tecido adiposo de 0,5 $\mathrm{cm}$ de espessura, expostas a feixes de raios $X$ típicos de mamografia gerados com altas voltagens de 28 a $32 \mathrm{kV}$ e combinação alvo/filtro Mo/Mo. Os coeficientes $D g N$ calculados para as mamas sem implante para um feixe de raios $\mathrm{X}$ de camada semirredutora $0,33 \mathrm{~mm} \mathrm{Al}$ apresentaram erro médio de $6,1 \%$ em relação aos obtidos na literatura, validando o modelo desenvolvido. Para as mamas com implantes, $D g N$ apresentou valores decrescentes em relação ao aumento da sua espessura e valores crescentes em relação ao aumento do raio do implante que variaram de $2 \%$ a $42 \%$. Doses glandulares médias totais $\left(D_{G}\right.$ total $)$ calculadas para o exame completo de 40 mulheres com implantes mamários apresentaram aumento médio de $7,2 \%$ (variando entre 0 e 18\%) quando comparadas com o modelo de Dance, que não inclui o implante. Isso indica que $D g N$ específicos devem ser disponibilizados para estimativas realísticas de $D_{G}$ em mamografia de mulheres com implantes.
\end{abstract}

Palavras-chave: mamografia; implante mamário; dose glandular normalizada; método Monte Carlo.

\begin{abstract}
In mammography of women with breast implants, four additional images with the posterior displacement of the silicone gel are necessary in addition to the four standard incidences. In this radiographic practice, the dosimetric quantity related to the risk of radiation-induced breast cancer is the mean glandular dose $\left(D_{G}\right)$, obtained from the incident air kerma (Ki) and the normalized glandular dose $(D g N)$ conversion coefficient between these quantities. This study aimed to: (a) investigate the behavior of DgN coefficients for breasts of different thicknesses with implants of varying volumes by means of the MCNPX software that uses Monte Carlo method; and (b) evaluate the impact of the new DgN coefficients on $D_{G}$ assessment for mammography of a sample of 40 women with implants. For this, it was simulated breasts with and without implants, composed of a homogeneous mixture of $50 \%$ adipose tissue and $50 \%$ glandular tissue wrapped in a layer of $0.5 \mathrm{~cm}$ thick adipose tissue. They were exposed to typical mammography X-ray beams generated with high voltages of 28 to $32 \mathrm{kV}$ and target/filter combination Mo/Mo. DgN coefficients for breasts without implants for an X-ray beam of $0.33 \mathrm{~mm}$ Al half value layer presented an average error of $6.1 \%$ in relation to those in the literature, validating the model used. For breasts with implants, DgN presented decreasing values in relation to increasing breast thickness and increasing values (from $2 \%$ up to $42 \%)$ in relation to increasing implant volume. $D_{G}$ calculated for the complete examination $\left(D_{G}\right.$ total $)$ of 40 women with breast implants showed an average increasing of $7.2 \%$ (between $0 \%$ and $18 \%$ ) when compared to the Dance model, which does not include the implant. This indicates that, specific DgN for this radiographic practice shall be available for realistic estimates of $D_{G}$ in mammography of women with implants.
\end{abstract}

Keywords: mammography; breast implant; normalized glandular dose; Monte Carlo method.

\section{Introdução}

$\mathrm{Na}$ mamografia de mulheres com implantes mamários, além das quatro incidências padrões (Figura 1a), são necessárias mais quatro com o deslocamento posterior do gel de silicone (Figura 1b). Nesta prática radiográfica, a grandeza dosimétrica relacionada ao risco de câncer radioinduzido na mama é a dose glandular média $\left(D_{G}\right)^{1}$, obtida a partir do kerma no ar incidente $\left(K_{i}\right)$ e do coeficiente de conversão dose glandular normalizada $(D g N)$ entre estas grandezas.

Tendo em vista que o exame radiográfico das mamas de mulheres portadoras de implantes mamários inclui um maior número de imagens, elas recebem doses maiores em relação às que não 
possuem ${ }^{2}$. Assim, torna-se relevante a estimativa da $D_{G}$ nas oito incidências do exame, principalmente nas quatro com a presença dos implantes mamários, e a consequente avaliação do risco para estas mulheres.

Figura 1 - Mamografias com: (a) presença dos implantes mamários; (b) deslocamento posterior do gel de silicone por meio da manobra de Eklund (b).

(a)
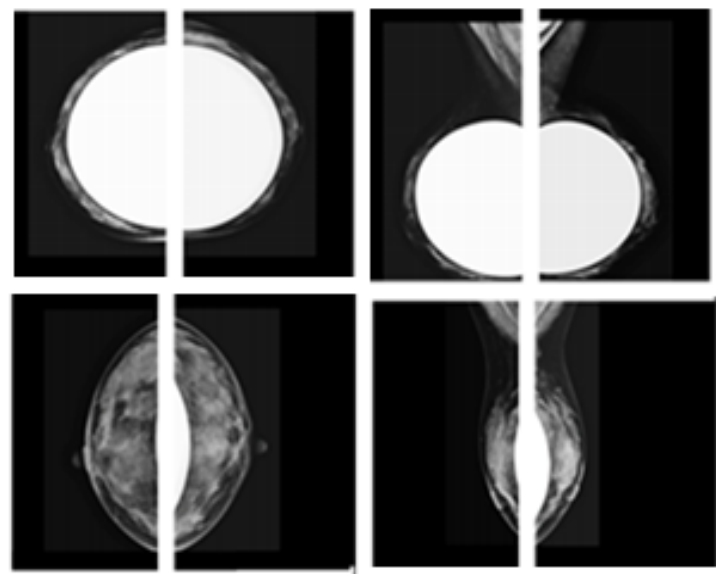

Fonte: Couto L S et al (2019)

Com equipamentos submetidos a um controle de qualidade contínuo e técnicas radiográficas otimizadas, este risco é considerado pequeno, mas é importante que seja minimizado. Assim, a avaliação da $D_{G}$ é um item importante do Programa de Garantia de Qualidade (PGQ) dos serviços de mamografia, permitindo que as técnicas radiográficas sejam otimizadas tanto sob o ponto de vista da dose, como da qualidade de imagem para o diagnóstico.

Os protocolos internacionais de garantia de qualidade ${ }^{3,4}$ adotam o modelo de cálculo da $D_{G}$ a partir do kerma no ar incidente $\left(K_{i}\right)$ e dos coeficientes de conversão estabelecidos por Dance e colaboradores $^{5-7}$, determinados por simulações matemáticas usando o método Monte Carlo. Este modelo de Dance não inclui implantes nas mamas simuladas nos cálculos dos coeficientes de conversão.

A revisão da literatura forneceu um único trabalho realizado por Beckett e Kotre ${ }^{8}$ no ano 2000 no qual os autores apresentam um modelo para a estimativa de $D_{G}$ em mamografia de mulheres com mamas aumentadas. Ele é baseado no acréscimo de volume da mama devido ao implante. Dance e Sechopoulus ${ }^{9}$ destacam, no entanto, que este estudo é de 2000 e, portanto, é baseado no sistema tela filme. Uma vez que o controle automático de exposição dos sistemas atuais de mamografia digital é baseado na espessura da mama comprimida (em alguns casos) e em uma exposição de teste pré-aquisição, os parâmetros de exposição selecionados seriam muito diferentes dos estudados por Becket e Kotre e, portanto, esta comparação de $D_{G}$ pode não ser aplicável à mamografia digital ${ }^{9}$.

O presente trabalho apresenta um novo modelo para o cálculo dos coeficientes de conversão do kerma no ar incidente para a dose glandular média em mamografia de mulheres com implantes mamários tendo como base a relação entre a área da imagem ocupada pelo implante e a área total da mama, incluindo o implante. Nele, é também avaliado o impacto dos novos coeficientes $D g N$ na estimativa da $D_{G}$ para mamografia digital de uma amostra de mulheres portadoras de implantes.

\section{Materiais e Métodos}

\subsection{Método Monte Carlo e Parâmetros de Exposição}

O método Monte Carlo foi utilizado para o cálculo da dose glandular média e o kerma no ar incidente por meio do software MCNPX. Os resultados das simulações são normalizados e apresentados individualmente para cada fóton. Para as simulações deste trabalho foram utilizados 1 milhão de fótons.

$O$ feixe de raios $X$ foi simulado de forma cônica, com o ponto focal situado no centro da geometria e à $60 \mathrm{~cm}$ da mama (Figura 2). As distribuições de probabilidade de emissão de fótons foram estimadas por meio de espectros de raios $X$ de mamografia de 28 a 32 kV e combinação alvo/filtro Mo/Mo, gerados pelo modelo MASMICS, desenvolvido por Hernandez et al $(2017)^{10}$.

Figura 2 - Geometria do feixe de raios $X$ e da mama com implante simulados para uso pelo MCNPX.

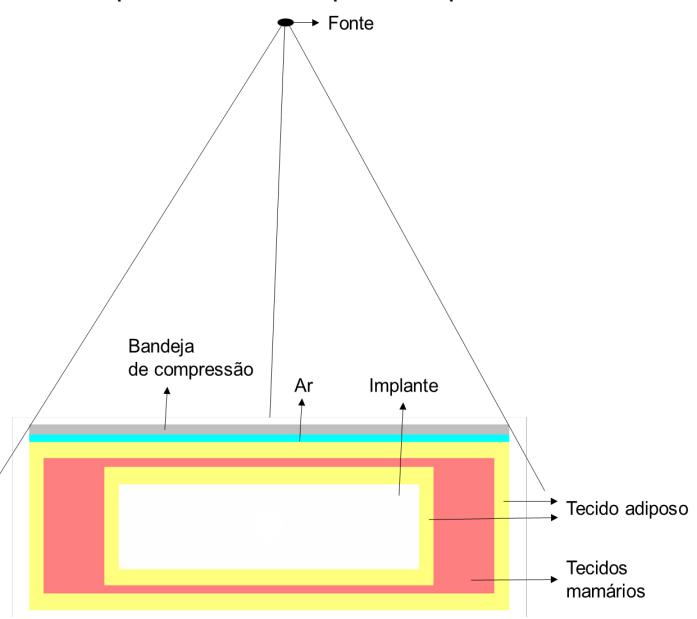

Parâmetros de definição de limites do campo de radiação foram utilizados para tornar retangular a área do feixe de fótons incidentes na mama, tal como nas simulações de Dance (1990) $)^{5-7}$.

\subsection{Geometria da Mama e Composição Elementar}

Os parâmetros geométricos das mamas usados nesse trabalho foram os mesmos utilizados por Dance $(1990)^{5-7}$. As mamas têm forma de um semicilindro com $8 \mathrm{~cm}$ de raio, $16 \mathrm{~cm}$ de comprimento e diversas espessuras totais, incluindo uma camada externa de $0,5 \mathrm{~cm}$ de tecido adiposo. Elas são compostas por uma mistura homogênea de $50 \%$ tecido adiposo e $50 \%$ tecido glandular. Foram utilizadas a composição e densidade da mama $50 \% / 50 \%$ apresentadas no trabalho de Hammerstein et al (1979) ${ }^{11}$. Foi inserido um implante mamário de silicone, na parte homogênea da mama, com formato semicilíndrico, raio variando entre 3,5 e $6,5 \mathrm{~cm}$ em intervalos de $0,5 \mathrm{~cm}$ e espessura igual a metade da 
espessura da mama. Também foi simulada sobre a mama uma placa compressora retangular de acrílico de $0,3 \mathrm{~cm}$ de espessura.

\subsection{Cálculo do Kerma no Ar Incidente}

O kerma no ar incidente foi calculado analiticamente pelo MCNPX utilizando os dados de fluência de fótons incidente por meio da equação (1):

$$
K_{i}=\sum \Phi(E) E\left(\frac{\mu_{t r}(E)}{\rho}\right)_{a r}
$$

Onde $\Phi(E)$ é a fluência de fótons na entrada da superfície da pele, $E$ é a energia de cada fóton do espectro e $\left(\frac{\mu_{t r}(E)}{\rho}\right)$ são os coeficientes mássicos de transferência de energia para o ar. Foi simulado um bloco de ar de 0,2 cm de espessura entre a mama e a placa compressora. A fluência de fótons no bloco de ar foi convertida para kerma no ar utilizando os fatores de conversão da publicação ICRP $51^{12}$.

Para evitar a presença de fótons retroespalhados no cálculo de kerma no ar incidente, foram retiradas todas as outras células da simulação, permanecendo apenas a célula de ar.

\subsection{Cálculo da Dose Glandular Média (Dg)}

A dose glandular média foi calculada utilizando a equação (2):

$$
D_{G}=\frac{N}{M_{g}} \sum_{j=1}^{N} E_{g}^{j}
$$

Onde $E_{g}^{j}$ é a energia depositada no tecido glandular por fóton, calculada pelo MCNPX, N é o número de fótons simulados, $M_{g}$ é a massa de tecido glandular presente na mistura homogênea. A Equação (2) foi aplicada tanto para o cálculo de $D_{G}$ para as mamas sem implantes como para as mamas com implantes. Nesse caso, a massa total de mistura homogênea foi reduzida em função inserção do implante.

\subsection{Coeficiente de Conversão Dose Glandular Normalizada (DgN)}

O coeficiente de conversão dose glandular normalizada foi obtido através da razão entre a dose glandular média e o kerma no ar incidente na superfície da mama, conforme a equação (3):

$$
D g N=\frac{D_{G}}{K_{i}}\left(\frac{m G y}{m G y}\right)
$$

Na prática da dosimetria em mamografia, a dose glandular média em uma exposição é determinada por meio da equação (4) através da medida do kerma no ar incidente e do uso do coeficiente de conversão dose glandular normalizada, $D g N$.

$$
D_{G}=K_{i} * D g N
$$

2.6. Dose Glandular Média Total (Dgtotal) para Mamografia de uma Amostra de Mulheres com Implantes

A dose glandular média total (DGtotal) em mamografia de mulheres com implante foi calculada como sendo a média da soma das $D_{G}$ das quatro incidências de cada mama, como mostrado pela equação (5).

$D_{\text {Gtotal }}=\left[\sum_{1}^{4} D_{G}\right.$ (mama dir. $)+\sum_{1}^{4} D_{G}$ (mama esq. $\left.)\right] / 2$

Os coeficientes $D g N$ calculados neste trabalho foram utilizados na determinação da $D_{\text {Gtotal }}$ de uma amostra de exames completos de 40 mulheres com composição mamária aproximada de 50\%/50\%, com o objetivo de comparação com a $D_{\text {Gtotal }}$ determinada pelo modelo de Dance $(1990)^{5-7}$. Com isso, foi possível avaliar o seu impacto na estimativa das doses recebidas pelas mulheres portadoras de implantes quando realizam mamografia.

\subsection{Questões Éticas e Legais}

As informações sobre os parâmetros técnicos empregados para a realização das mamografias da amostra de exames utilizados neste trabalho foram obtidas nos cabeçalhos DICOM das imagens, nas quais foram suprimidos os dados de identificação das mulheres. O projeto foi aprovado pelo comitê de ética do Hospital das Clínicas da UFG sob $n^{\circ} \mathrm{CAAE}$ : 56513416.0.0000.5078.

\section{Resultados}

\subsection{Coeficientes de conversão DgN para mamas sem implantes}

Para a validação do modelo desenvolvido neste trabalho, $D g N$ foi determinado para mamas sem implantes de espessuras de $2 \mathrm{~cm}$ a $8 \mathrm{~cm}$ quando expostas a um feixe de raios $X$ gerado pelo software MASMICS com tensão de 28 kV e combinação alvo de Mo e filtro de Mo de $30 \mu \mathrm{m}$ de espessura. A camada semirredutora (HVL) do feixe resultante foi de $0,33 \mathrm{~mm}$ de alumínio. A Figura 3 mostra os resultados obtidos quando comparados com os de Dance $^{5-7}$ para a mesma HVL.

Figura 3 - Comparação dos coeficientes de conversão DgN em função da espessura da mama para o feixe de $28 \mathrm{kV}$ e Mo/Mo calculados por Dance ${ }^{5-7}$ e por deste trabalho para mamas sem implante.

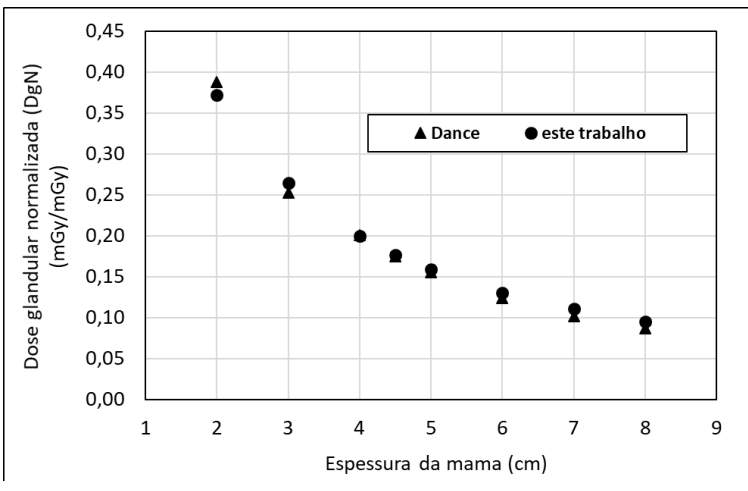


Para diversas espessuras de mama sem implantes, os $D g N$ determinados pelo modelo desenvolvido neste trabalho apresentaram um erro médio de $6,1 \%$ quando comparados com o modelo de Dance D-7. $^{5-1}$.

\subsection{Coeficientes de conversão DgN para mamas com implantes}

Os coeficientes de conversão $D g N$ foram calculados para as mamas de espessuras de $5 \mathrm{~cm}$ a $10 \mathrm{~cm}$, com intervalos de $1 \mathrm{~cm}$, quando inseridos implantes mamários de raios de $3,5 \mathrm{~cm}$ a $6,5 \mathrm{~cm}$, com intervalos de $0,5 \mathrm{~cm}$. Após as simulações, foram obtidos os resultados mostrados na Figura 4 para o feixe de $28 \mathrm{kV}$ e Mo/Mo. Para este feixe de raios $\mathrm{X}$ a presença dos implantes mamários resultou em aumento de $D g N$ variando, quando comparado com o modelo de Dance ${ }^{5-7}$, de $2 \%$ para mama de $5 \mathrm{~cm}$ de espessura com implante de $3,0 \mathrm{~cm}$ de raio até $42 \%$ para a mama de $10 \mathrm{~cm}$ de espessura com implante de $6,5 \mathrm{~cm}$ de raio.

Figura 4 - Coeficientes $D g N$ em função da espessura da mama e do raio do implante para o feixe de $28 \mathrm{kV}$ e Mo/Mo.

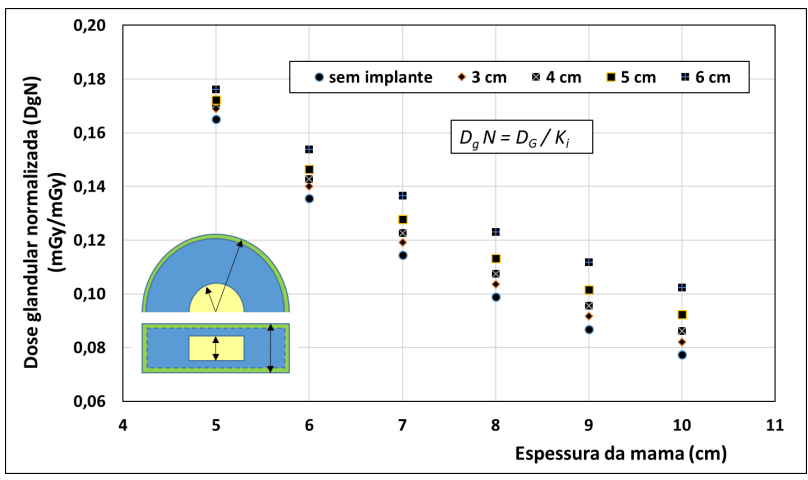

\subsection{Coeficientes de conversão $D g N$ em função da} razão entre a área do implante e a área da mama

Neste trabalho também foi investigado o comportamento de $D g N$ em função da razão entre a área do implante e a área da mama. A Figura 5 mostra os resultados calculados para mamas de diferentes espessuras e razões área do implante/área da mama para o feixe de $28 \mathrm{kV}$ e Mo/Mo.

Figura 5 - Coeficientes $D g N$ em função da razão da área do implante/área da mama para mamas de diferentes espessuras para o feixe de $28 \mathrm{kV}$ e Mo/Mo.

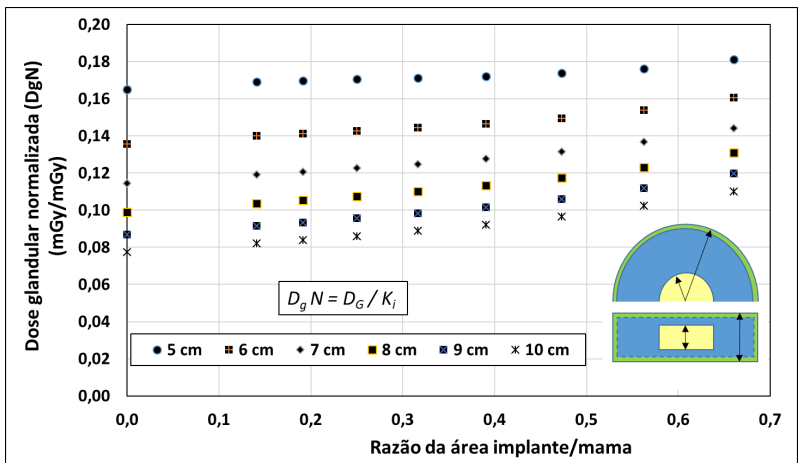

Foi verificado que, para mamas de todas as espessuras, quanto maior a razão, maior é o coeficiente de conversão dose glandular normalizada. Com esses dados, nas exposições com a técnica radiográfica de $28 \mathrm{kV}$ e Mo/Mo, os coeficientes $D g N$ para mamas com implantes entre as espessuras de $5 \mathrm{~cm}$ a $8 \mathrm{~cm}$ quando comprimidas podem ser calculados com facilidade bastando que se conheça a espessura da mama comprimida e a razão entre a área do implante e a área da mama. Para tal, devem ser usados polinômios de segundo grau, cujos coeficientes de ajuste são mostrados na Quadro 1.

Quadro 1 - Coeficientes de ajuste para o cálculo de $D g N$ através da razão entre a área do implante / área da mama, para diferentes espessuras de mama e para o feixe de $28 \mathrm{kV}$ e Mo/Mo.

\begin{tabular}{|c|c|c|c|c|c|c|}
\hline \multicolumn{7}{|c|}{ Coeficientes de ajuste pela razão do área do implante /área da mama* } \\
\hline \multirow{2}{*}{ Coeficientes de ajuste } & 5 & 6 & 7 & 8 & 9 & 10 \\
\cline { 2 - 7 } & 0.0408 & 0.0518 & 0.0535 & 0.0537 & 0.0525 & 0.0501 \\
\hline $\mathrm{a}$ & -0.0113 & -0.0036 & 0.0038 & 0.0083 & 0.0108 & 0.0130 \\
\hline $\mathrm{b}$ & 0.1703 & 0.1400 & 0.1180 & 0.1018 & 0.0894 & 0.0795 \\
\hline $\mathrm{C}$ & 0.98 & 0.99 & 1.00 & 1.00 & 1.00 & 1.00 \\
\hline $\mathrm{R}^{2}$ & onde $\mathrm{x}$ é a razão da área do implante / área da mama \\
\hline$* \mathrm{y}(\mathrm{x})=\mathrm{ax}^{2}+\mathrm{bx}+\mathrm{c}$
\end{tabular}

3.4. Impacto dos novos DgN na dose glandular média total ( $D_{\text {Gtotal }}$ ) para a mamografia de uma amostra de mulheres com implantes

A dose glandular média total ( $\left.D_{\text {Gtotal }}\right)$ em mamografia de uma amostra de 40 mulheres com implante foi calculada por meio da razão entre a área do implante e a área da mama, dos $K i$ medidos e $D g N$ determinados neste trabalho para os feixes de raios $\mathrm{X}$ de 28 a $32 \mathrm{kV}$ e Mo/Mo. As razões área do implante/área da mama foram determinadas a partir de informações extraídas das imagens das pacientes e com o auxílio do software ImageJ. A D $D_{\text {Gtotal }}$ para estas mulheres foi também calculada usando os mesmos valores de $K i$ e os $D g N$ obtidos pelo modelo de Dance $(1990)^{5-7}$. A Figura 6 mostra as distribuições de $D_{\text {Gtotal }}$ calculadas para os dois modelos.

Figura 6 - Distribuição das $D_{\text {Gtotal para } 40 \text { mulheres }}$ portadoras de implantes usando os coeficientes de conversão de Dance et al (2000) e os deste trabalho.

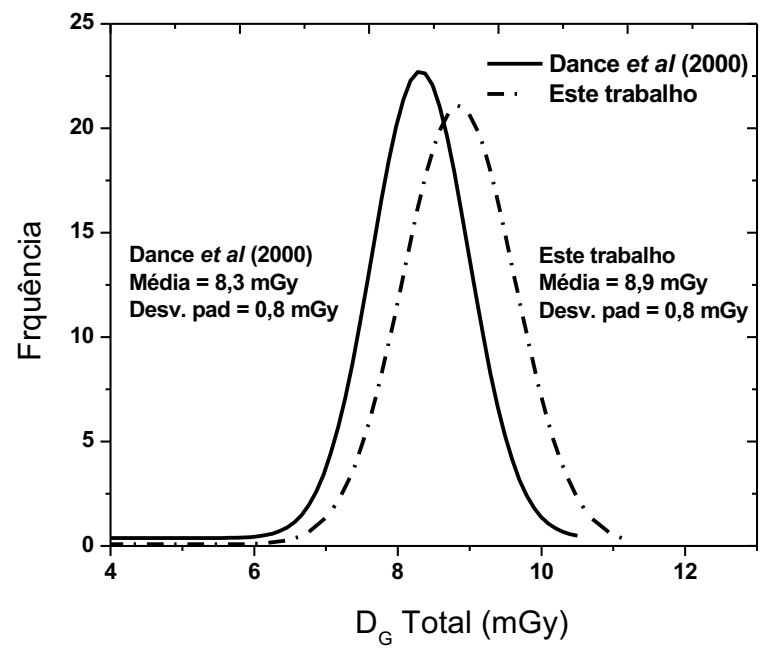


Pode ser visto na Figura 6 que a dose glandular média total recebida pela amostra de 40 mulheres com implantes mamários usadas para comparação com o método de Dance (1990) $)^{5-7}$, apresentou aumento médio estatisticamente significativo ( $p<$ 0,001 ) de $7,2 \%$ (variando entre 0 e $18 \%$ ) quando comparadas com o modelo de Dance, que não inclui o implante.

\section{Discussão}

Os resultados deste trabalho impactam significativamente nas estimativas da dose glandular média em mamografia de mulheres com implante. $\mathrm{O}$ modelo atual dos protocolos de dosimetria e dos softwares dos mamógrafos digitais DR utilizam, na sua maioria, o modelo de Dance $(1990)^{5-7}$, que não inclui os implantes nas mamas simuladas para a determinação dos coeficientes de conversão do kerma no ar incidente para a dose glandular média.

O modelo de cálculo de $D g N$ neste trabalho apresentou uma diferença média de $6,1 \%$ em relação aos resultados do modelo de mama sem implante de Dance $(1990)^{5-7}$. As diferenças entre os dois modelos de cálculo podem ser atribuídas, principalmente, aos espectros de raios $X$ utilizados nas simulações e às diferentes versões do software MCNPX utilizados. Dance ${ }^{5-7}$ utilizou 90 espectros diferentes para simular os fatores de conversão e uma versão de 1990 do software.

Com a inclusão dos implantes na simulação, foi observado, para o feixe de raios X de $28 \mathrm{kV}$ e Mo/Mo, um aumento nos coeficientes de conversão em função da espessura da mama comprimida e do raio do implante de até $42 \%$, como mostrado na Figura 4. Calculando a dose glandular média total de uma amostra de 40 mulheres portadoras de implante mamário e considerando a razão área do implante/área da mama foi observado um aumento de até $18 \%$ em relação a estimativa de $D_{\text {Gtotal }}$ quando utilizado o modelo de Dance (1990) ${ }^{5-7}$. Estes resultados discordam dos apresentados por Beckett e Kotre $(2000)^{8}$ que determinaram $D_{G}$ em mamografia de mulheres portadoras de implantes similares ou menores do que em mamografia de mulheres sem implantes mamários, levando em conta as técnicas radiológicas utilizadas.

\section{Conclusões}

Os resultados deste trabalho indicam que, para estimativas realísticas de $D_{G}$ em mamografia de mulheres com implantes, $D g N$ específicos para esta prática radiográfica devem ser disponibilizados. Com base nestas estimativas mais realísticas das doses glandulares, poderão ser realizados estudos de otimização das técnicas radiográficas tanto sob o ponto de vista da dose para a redução do risco associado ao exame, como da qualidade de imagem para o diagnóstico mais preciso, aumentando o benefício para as mulheres portadoras de implantes mamários.

\section{Agradecimento}

Ao CNPQ pelo apoio ao projeto de iniciação científica sob o número de processo 129770/2019-1. Também agradecem a Dra. Rosângela Silveira Corrêa pela organização dos parâmetros técnicos empregados para a realização das mamografias da amostra de mulheres utilizada neste trabalho.

\section{Referências}

1. National Research C. Health Risks from Exposure to Low Levels of Ionizing Radiation: BEIR VII Phase 2. Washington, DC: The National Academies Press; 2006.

2. Couto LS, Freitas-Junior R, Correa RS, Peixoto JE, Almeida $\mathrm{CD}$, Rodrigues DCN, et al. Mean glandular dose in digital mamography in women with breast implants. Journal of Radiological Protection. 2019;39(2):498-510.

3. IAEA - International Atomic Energy Agency. Quality assurance programme for digital mammography. Human health series No. 17. Viena: 2011.

4. Perry N, Broeders M, de Wolf C, Tornberg S, Holland R, Von Karsa R. European Guidelines for Quality Assurance in BreastCancer Screening and Diagnosis. 4th ed. Luxembourg: Luxembourg office for official publications of the European Communities, 2006.

5. Dance DR. Monte-Carlo calculation of conversion factors for the estimation of mean glandular breast dose. Physics in Medicine and Biology. 1990;35(9):1211-9.

6. Dance DR, Skinner CL, Young KC, Beckett JR, Kotre CJ. Additional factors for the estimation of mean glandular breast dose using the UK mammography dosimetry protocol. Physics in Medicine \& Biology. 2000;45(11):3225.

7. Dance DR, Young KC, Van Engen RE. Further factors for the estimation of mean glandular dose using the United Kingdom, European and IAEA breast dosimetry protocols. PHYS MED BIOL. 2009;54(14):4361-72.

8. Beckett JR, Kotre CJ. Estimation of mean glandular dose for mammography of augmented breasts. Physics in Medicine and Biology. 2000;45(11):3241-52.

9. Dance DR, Sechopoulos I. Dosimetry in x-ray-based breast imaging. Physics in Medicine and Biology. 2016;61(19): R271R304.

10. Hernandez AM, Seibert JA, Nosratieh A, Boone JM. Generation and analysis of clinically relevant breast imaging $\mathrm{X}$ ray spectra. Medical Physics. 2017;44(6):2148-60.

11. Hammerstein RG, Miller DW, White DR, Ellen Masterson M, Woodard HQ, Laughlin JS. Absorbed Radiation Dose in Mammography. Radiology. 1979;130(2):485-91.

12. ICRP. Statement from the 1987 Como meeting of the ICRP. ICRP Publication 51. Oxford: Pergamon; 1987.

\section{Contato:}

Josilene Cerqueira Santos

Instituto de Radioproteção e Dosimetria/CNEN

Av. Salvador Allende, 3773 - Rio de Janeiro (RJ)

E-mail: josilene@usp.br

Alexander Camargo Firmino da Silva

Instituto de Radioproteção e Dosimetria/CNEN

Av. Salvador Allende, 3773 - Rio de Janeiro (RJ)

E-mail: alexander.camargo@oi.com.br

Lilian S. Couto

Programa de Mastologia, Universidade Federal de Goiás, Goiânia(GO)

$1^{\text {a }}$ Avenida, S. Leste Universitário, Goiânia (GO)

E-mail: liliancouto@hotmail.com

João Emílio Peixoto

Instituto Nacional de Câncer -INCA

Rua do Rezende, 128, Rio de Janeiro (RJ)

E-mail: joao.peixoto@inca.gov.br 